Article

\title{
Time Dependence of Wetting Behavior Upon Applying Hierarchic Nano-Micro Periodic Surface Structures on Brass Using Ultra Short Laser Pulses
}

\author{
Stefan Rung ${ }^{1, *}$, Simon Schwarz ${ }^{1}$, Babette Götzendorfer ${ }^{1}$, Cemal Esen ${ }^{2}$ and Ralf Hellmann ${ }^{1}$ \\ 1 Applied Laser and Photonics Group, University of Applied Sciences Aschaffenburg, Würzburger Straße 45, \\ 63743 Aschaffenburg, Germany; Simon.schwarz@h-ab.de (S.S.); Babette.Goetzendorfer@h-ab.de (B.G.); \\ ralf.hellmann@h-ab.de (R.H.) \\ 2 Applied Laser Technologies, Ruhr-Universität Bochum, Universitätsstraße 150, 44801 Bochum, Germany; \\ esen@lat.rub.de \\ * Correspondence: stefan.rung@h-ab.de; Tel.: +49-6022-81-3694
}

Received: 28 March 2018; Accepted: 25 April 2018; Published: 2 May 2018

\begin{abstract}
We present a comprehensive experimental study on laser-induced hierarchic nano-micro periodic surface structures on brass that influences wetting behavior. Using ultra short laser pulses with a wavelength of $1030 \mathrm{~nm}$, large scaled areas completely covered by laser-induced periodic surface structures (LIPSS) are generated with these areas being superimposed by ablation trenches and u-ripples. The influence of the incident laser fluence and pulse overlap on the apparent contact angle for coverage of the surface with distilled water with a surface tension of $74 \mathrm{mN} / \mathrm{m}$ are examined with its temporal evolution being observed over a period of two weeks. Our results show an initial drop in the apparent contact angle below the angle of an unstructured surface. Using atomic force microscopy, the roughness factor described by the Wenzel model is determined and compared to the roughness factor given by the apparent contact angle measurement. The ascertained difference in roughness cannot be entirely attributed to the topography of the laser-structured surface. We suggest that changes in the surface chemistry additionally alter the wetting behavior as confirmed by X-ray photoelectron spectroscopy (XPS) measurements. On a time scale of days after laser irradiation, the apparent contact angle increases into the hydrophobic range. Both the absolute apparent contact angle and this temporal change reveal a pronounced dependence on the applied laser fluence and pulse overlap. In particular, increasing both, the fluence and the pulse overlap leads to smaller apparent contact angles directly after the irradiation and to higher apparent contact angles after an observation period of two weeks.
\end{abstract}

Keywords: wettability; time dependence; laser-induced periodic surface structures

\section{Introduction}

Over the last decade, the increased commercial availability of industrial grade ultra short pulsed laser systems has fostered many applications of this innovative laser source. Beside the advantage of non-thermal material processing, new approaches for surface structuring have been intensively studied. One of these novel applications is the generation of laser-induced periodic surface structures (LIPSS), which were firstly observed in 1965 by Birnbaum [1]. Generally, LIPSS are separated in two common types. Low spatial frequency LIPSS (LSFL) appear with a spatial periodicity near the incidence laser wavelength, whereas high spatial frequency LIPSS (HSFL) exhibit a spatial periodicity clearly below the laser wavelength [2]. For metals, the orientation of LSFL is perpendicular and that of HSFL is parallel to the polarization direction of the incident laser radiation. The origin of LSFL is described as the interaction of the electromagnetic field of the laser and a surface scattered wave 
generated by the incident laser light. This surface scattered wave forms the basis of the so-called efficacy factor theory, which is associated with surface plasmon polaritons (SPP) [3]. This model of the origin explains the direction and the spatial wavelength of LSFL on metals. Recent studies suggest that another type of periodic structure, often referred to as unclassical ripples, u-ripples, or grooves, with a spatial period much greater than the incident laser wavelength, may appear during ultra-short pulsed laser irradiation. The orientation of the latter is found to be parallel to the polarization of the laser beam [4-6]. The formation of such u-ripples has been attributed to capillary waves by an inhomogeneous temperature distribution as a result of scattered laser pulses on a molten surface layer [5]. With respect to the manifold potential applications of periodically structured surfaces, the aforementioned types of LIPSS have been intensively investigated on metals $[7,8]$, semiconductors [3,4], and dielectrics [9-11]. Specifically, periodic surface structures can be used for controlling cell growth in biotechnology [12], friction and wear optimization [13], testing of mechanical properties [14], and influencing the emission properties of $\mathrm{THz}$ transmitters [15]. In particular, the influence of LSFL on the wetting properties of surfaces can be applied in microfluidics [16] and to self-cleaning components [17]. Commonly, Ti/sapphire lasers [18-20] or nanosecond lasers [21-24] are used in scientific reports to modify the wetting behavior of surfaces; however, for industrial applications, it is desirable to use femtosecond lasers with a high repetition rate, enabling high throughput and process efficiency. There are fundamentally three different states of wetting behavior differentiated with respect to the contact angle, namely hydrophilic $\left(\Theta<90^{\circ}\right)$, hydrophobic $\left(\Theta>90^{\circ}\right)$, and super-hydrophobic $\left(\Theta>150^{\circ}\right)$. These states can be practically realized either by an additional deposited function layer or by structuring the surface to modify the surface roughness in a specific manner. Since the generation of LIPSS depends sensitively on the number of laser pulses per spot and the applied laser pulse energy, a comprehensive study of the influencing factors is indispensable as to control the achievable wetting properties for the targeted application, e.g., hydrophilic states for microfluidics or super hydrophobic states for self-cleaning surfaces.

In this study, we report on the generation of combined LSFL, u-ripples, and ablation trenches on brass and analyze the wetting behavior immediately after laser irradiation and its temporal evolution on a time scale of several days. Whereas the apparent contact angle initially reveals a pronounced drop as compared to unstructured brass, it increases into the hydrophobic regime and saturates over several days. This temporal evolution has already been shown for several metallic surfaces after femtosecond-based laser structuring $[17,25-28]$ and is mainly described by a highly chemical reactive surface direct after laser treatment and a growing passive hydrophobic layer rich on carbon from the composition of $\mathrm{CO}_{2}$ [29]. Both the initial drop and the subsequent increase reveal a strong dependence on pulse overlap and laser fluence. For a further understanding of the initial drop of the apparent contact angle, the wetting behavior according to the Wenzel model [30] is discussed with the roughness factor being determined by atomic force microscope measurements. Our results imply that the change in wetting behavior cannot entirely be attributed to the laser-induced changes in the topography, and we suggest that changes in the surface element concentration additionally alter the wetting behavior, namely, by an increase in the oxygen concentration and a change in the concentration ratio between carbon and tin/copper, as confirmed by XPS measurements of unstructured reference samples and laser-structured specimens. The novel approach of this study is the combination of the high pulse repetition rate, the fs-pulse duration, and the $1030 \mathrm{~nm}$ laser wavelength with comprehensive consideration of laser fluence, pulse overlap, and time-based effects on the apparent contact angle appearance of water on flat brass surfaces. The laser-based hierarchic nano and micro structures are analyzed, and the resulting surface advance is compared to the apparent contact angle measurement to identify the chemical modification directly after laser treatment. The results can help to implement hierarchic nano and micro structures into surface engineering approaches. 


\section{Experimental}

We used an ultra short pulsed laser (Pharos 10-600-PP, Light Conversion, Vilnius, Lithuania) with a pulse duration of $220 \mathrm{fs}(\mathrm{FWHM})$ at a repetition rate of $50 \mathrm{kHz}$. For the generation of the microand nanostructures, we used the fundamental emission wavelength of $1030 \mathrm{~nm}$. Figure 1 shows the experimental setup for the surface treatment. The energy of the laser beam was adjusted by an external attenuator. With a half wave plate in front of the focusing unit, the linear polarization of the laser beam was rotated parallel to the onwards used scanning direction. A galvo scanner (RTA AR800 $2 \mathrm{G}+$, Newson, Dendermonde, Belgium) was used in combination with a telecentric lens $(f=100 \mathrm{~mm})$ to focus the beam onto the sample with a spot diameter of $38 \mu \mathrm{m}\left(1 / e^{2}\right)$. LSFL were generated on $0.75 \mathrm{~mm}$ thick commercial available flat brass (CW 508L, CuZn37), which was cut in square pieces with a size of $16 \times 16 \mathrm{~mm}^{2}$. The pieces were ground with a multi-directional polishing machine (LS3V, REMET, Bologna, Italy) with several abrasive strengths and afterwards polished with suspensions down to a grain size of $1 \mu \mathrm{m}$ to achieve a reference surface with a roughness $R_{a}$ between $9 \mathrm{~nm}$.

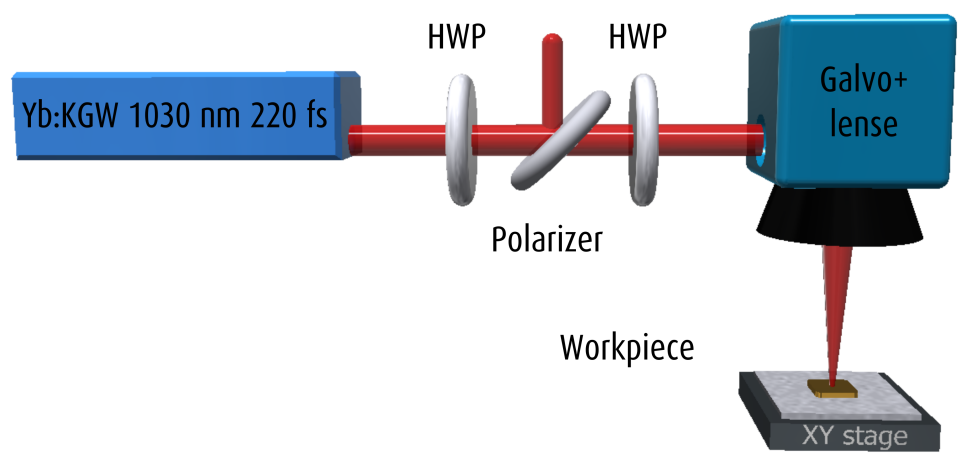

Figure 1. Schematic laser system setup with Light Conversion Pharos 10-600. First, the half wave plate (HWP) and Polarizer allow energy adjustment; second, the HWP controls the orientation of the laser-induced periodic surface structures (LIPSS).

To determine their influence on the generation of LIPSS, the fluence, i.e., the ratio of laser pulse energy and spot area, was varied between $0.87 \mathrm{~J} / \mathrm{cm}^{2}$ and $1.96 \mathrm{~J} / \mathrm{cm}^{2}$, and the scanning speed between $16 \mathrm{~mm} / \mathrm{s}$ and $144 \mathrm{~mm} / \mathrm{s}$, respectively. The latter correspond to effective pulse overlaps between $92 \%$ and $99 \%$. For each parameter combination, the scanning speed was set to achieve comparable track energies for each fluence (namely 2.5, 5, 7.5, and $10 \mathrm{~J} / \mathrm{m}$ have been employed). While LSFL and u-ripples generated self-organized periodic structures that appear even for static laser illumination, ablated lines were generated in a periodic fashion by a scanning path that aligned individual lines next to each other. Becausethe ablation width increased with increasing fluence and track energy, the distance between individual ablation lines had to be adjusted for each parameter set in order to avoid damage in between lines and to ensure complete coverage of the surface with a periodic ablation pattern. Therefore, in the experimental evaluation, we also determined a periodicity for the ablated lines $\left(\Lambda_{\text {ablation, }}\right.$, see Table 1$)$, and this periodicity was in turn used in the numerical analysis (Section 3.3).

For each parameter combination, three equal samples with structured fields of $4.5 \times 4.5 \mathrm{~mm}^{2}$ were generated. The experimental results of the apparent contact angle measurements, as shown in the following diagrams, included the determined mean values and standard deviations. The geometrical dimensions of $\Lambda_{L S F L}$ and $\Lambda_{u \text {-ripple }}$ were captured via Scanning Electron Microscopy (Phenom ProX, Phenom World, Eindhoven, The Netherlands) with subsequently picture analysis via 2D Fourier transformation. This approach figures the spatial frequency, the orientation, and the homogeneity of the generated LIPSS [11]. To evaluate the modulation depth of the LSFL and u-ripples, an atomic force microscope (Dimension Icon, Bruker Corporation, Billerica, MA, USA) in PeakForce Tapping Mode and for the determination of the modulation depth of the line ablation, a 3D optical microscope based 
on white light interferometry (Contour GT-K, Bruker Corporation) were used. The wetting behavior was investigated using a contact angle measurement system (OCA 25, Data Physics, Filderstadt, Germany). Using a dispensing unit, a droplet $(6 \mu \mathrm{L})$ of distilled water with a surface tension of $74 \mathrm{mN} / \mathrm{m}$ was deposited on the examined surface. A camera captures the droplet on the surface via the sessile drop method. The resulting image was analyzed to determine the apparent contact angle by the circular shape fitting of the drop. Studies by Kietzig et al. [29] and Martínez-Calderon et al. [25] have shown that the apparent contact angle of water on laser-structured surfaces depends on the elapsed time between the structuring process itself and the actual measurement. To evaluate this effect specifically on LIPSS, for each laser parameter set, three identical samples were initially produced, allowing measurements of the apparent contact angle over days on fresh, i.e., previously unused, specimens. Until the apparent contact angle was measured, all samples were stored under ambient air at a temperature of approximately $23^{\circ} \mathrm{C}$. XPS measurements have been performed with an ESCA spectrometer (Perkin Elmer) using monochromatic Al-K- $\alpha$ radiation providing quantitative information on the surface element concentration. High resolution measurements of detailed spectra resolving changes in the binding conditions are performed using an $\mathrm{Mg}-\mathrm{K}-\alpha$ cathode ray tube.

Table 1. Spatial wavelength and modulation depth of LSFL, u-ripples, and ablations in the dependence of applied laser fluence (rows) and track energy (columns). The values represent the mean of three measured specimens.

\begin{tabular}{|c|c|c|c|c|}
\hline & \multicolumn{4}{|c|}{ Spatial Wavelength $\Lambda_{L S F L} / \mathrm{nm}$} \\
\hline & $2.5 \mathrm{~J} / \mathrm{m}$ & $5 \mathrm{~J} / \mathrm{m}$ & $7.5 \mathrm{~J} / \mathrm{m}$ & $10 \mathrm{~J} / \mathrm{m}$ \\
\hline $0.87 \mathrm{~J} / \mathrm{cm}^{2}$ & 905 & 902 & 886 & 879 \\
\hline $1.63 \mathrm{~J} / \mathrm{cm}^{2}$ & 901 & 894 & 884 & 872 \\
\hline \multirow[t]{3}{*}{$1.96 \mathrm{~J} / \mathrm{cm}^{2}$} & 897 & 891 & 882 & 869 \\
\hline & \multicolumn{4}{|c|}{ Spatial Wavelength $\Lambda_{u-r i p p l e} / \mathrm{nm}$} \\
\hline & $2.5 \mathrm{~J} / \mathrm{m}$ & $5 \mathrm{~J} / \mathrm{m}$ & $7.5 \mathrm{~J} / \mathrm{m}$ & $10 \mathrm{~J} / \mathrm{m}$ \\
\hline $0.87 \mathrm{~J} / \mathrm{cm}^{2}$ & 1316 & 1557 & 1788 & 1808 \\
\hline $1.63 \mathrm{~J} / \mathrm{cm}^{2}$ & 1247 & 1316 & 1733 & 1812 \\
\hline \multirow[t]{3}{*}{$1.96 \mathrm{~J} / \mathrm{cm}^{2}$} & 1280 & 1339 & 1709 & 1800 \\
\hline & \multicolumn{4}{|c|}{ Spatial Wavelength $\Lambda_{\text {ablation }} / \mu \mathrm{m}$} \\
\hline & $2.5 \mathrm{~J} / \mathrm{m}$ & $5 \mathrm{~J} / \mathrm{m}$ & $7.5 \mathrm{~J} / \mathrm{m}$ & $10 \mathrm{~J} / \mathrm{m}$ \\
\hline $0.87 \mathrm{~J} / \mathrm{cm}^{2}$ & 25.8 & 27.8 & 28 & 28.4 \\
\hline $1.63 \mathrm{~J} / \mathrm{cm}^{2}$ & 24.5 & 29.6 & 32.1 & 31.2 \\
\hline $1.96 \mathrm{~J} / \mathrm{cm}^{2}$ & 25.2 & 33 & 32.3 & 33.2 \\
\hline
\end{tabular}

\section{Results and Discussion}

\subsection{Modulation Length and Depth Analysis of Laser-Structured Surfaces}

Figure 2 schematically shows the appearance of the laser-driven structures. The laser scanning direction is along the $X$-axis, so the height modulation is along the $Y$-axis. Even along the $Y$-axis, the u-ripple modulation occurs after femtosecond laser irradiation. Perpendicular to these structures, the LSFL appears along the $X$-axis. To visualize these structures, Figure 3 shows the identical area of a laser-structured brass surface for two measurement modes (compositional and topographical mode) of the SEM, generated by different SEM detector settings (Figure 3a,c), highlighting differently generated LSFL and u-ripples using a fluence of $0.87 \mathrm{~J} / \mathrm{cm}^{2}$ and a pulse overlap of $98.8 \%$. Whereas in Figure $3 \mathrm{a}$ LSFL can be seen with a periodicity of $879 \mathrm{~nm}$, i.e., slightly below the laser wavelength, Figure 3c 
depicts u-ripples in the same field of view, being perpendicularly oriented to the LSFL and having a periodicity of $1808 \mathrm{~nm}$. Performing a 2D Fourier analysis on these images yields information on the spatial frequency, orientation, and homogeneity of the periodic structures (Figure $3 b, d$ ). Please note that the above given figures for the periodicities are taken from the position of the centroids of the 2D Fourier spectra.
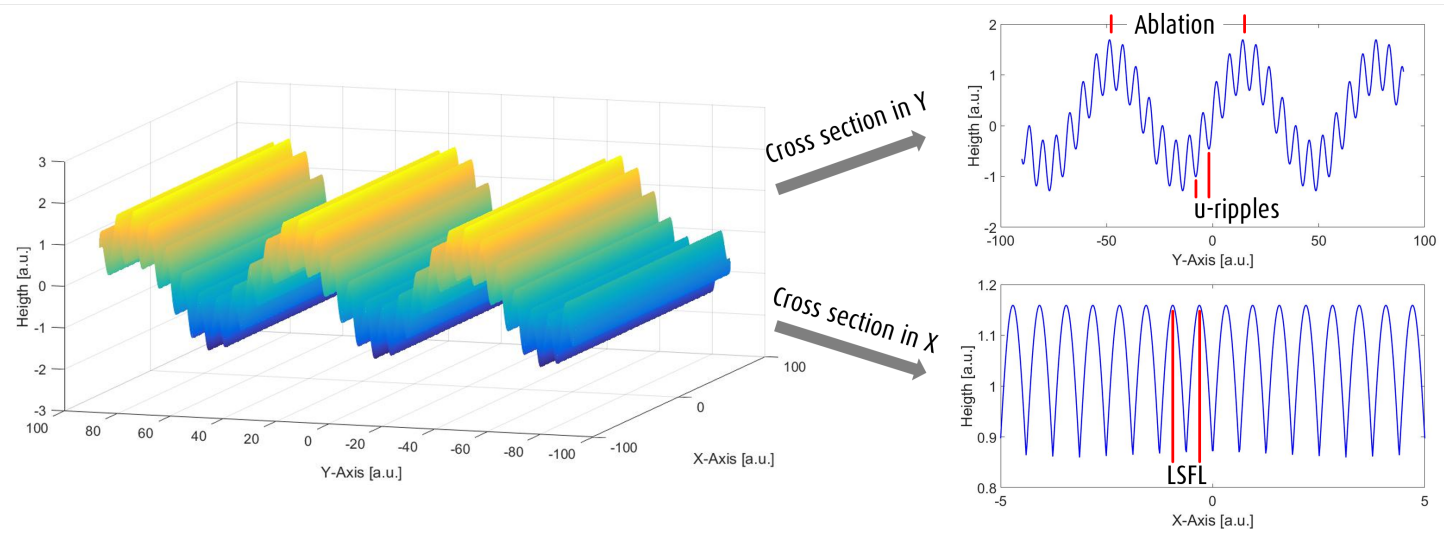

Figure 2. A schematic surface model of the laser-generated hierarchic periodic nano-micro structures showing both laser-ablated trenches with superimposed u-ripples and LIPSS perpendicular to the trenches and ripples. Please note the different scale for the LIPSS which appear on a nano-micro scale whereas ablation trenches and u-ripples are on a micro-scale.

Table 1 summarizes the measured periodicities and modulation depths of LSFL, u-ripples, and ablation lines as a function of the applied laser fluence and track energies, respectively. Apparently, in the studied parameter range, the LSFL period $\Lambda_{L S F L}$ decreases significantly with increasing track energy, i.e., decreasing scanning speed, and decreases slightly with increasing fluence. The decreasing spatial wavelength of the LSFL can be explained by the induction of a surface-plasma wave through the parametric decay of laser light [31]. The increasing pulse number is a result of a feedback mechanism based on a grating assisted SSP excitation and the incident laser radiation [4]. This behavior has similarly been reported for the generation of LIPSS on titanium by Golosov et al. [32], on silicon by Bonse et al. [4], and on titanium, molybdenum, platinum, and tungsten by Okamuro et al. [31]. Contrary to these trends of $\Lambda_{L S F L}$, the spatial periodicity of the u-ripples $\left(\Lambda_{u \text {-ripple }}\right)$ increases with increasing track energy and exhibits similarly small decreases with increasing fluence $[5,33]$. Finally, since the ablated line spatially extends as track energy and fluence increases, the periodicity of the ablated lines $\left(\Lambda_{\text {ablation }}\right)$ also increases according to the readjustment of the scanning distance between ablated lines (see Section 2).The modulation depths reveals a similar behavior for all three types of periodic structuring. With increasing track energy, i.e., increasing local energy deposition, $d_{L S F L}$, $d_{u \text {-ripple }}$, and $d_{\text {ablation }}$ increase, e.g., for $d_{L S F L}$, from about 160 to $260 \mathrm{~nm}$, for $d_{u \text {-ripple }}$, from about 200 to $350 \mathrm{~nm}$, and for $d_{\text {ablation, }}$, from 0.6 to $2.1 \mu \mathrm{m}$, respectively. With increasing fluence, however, the modulation depth remains almost constant on the respective level determined by the track energy. 


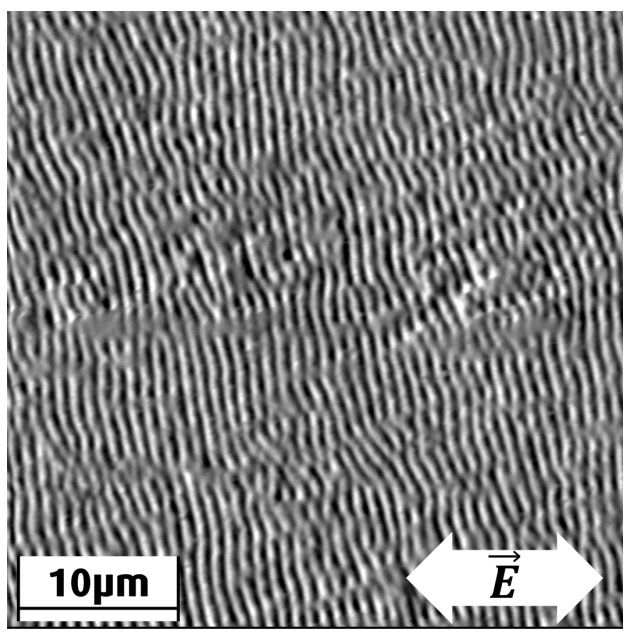

(a) LSFL

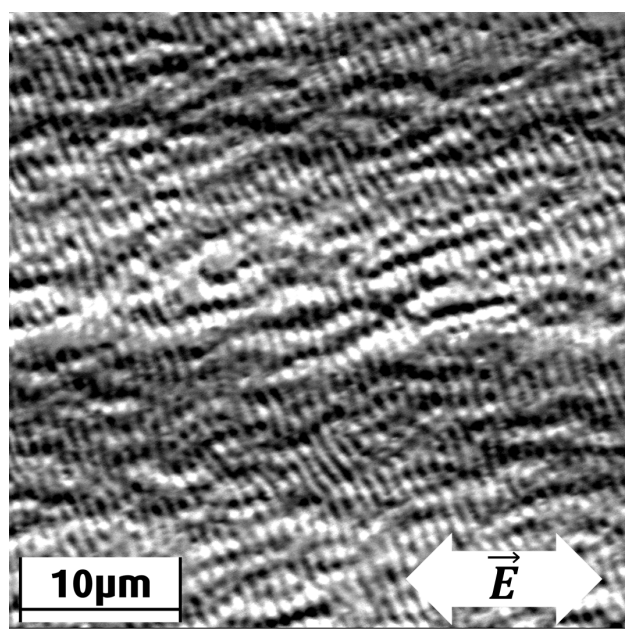

(c) u-ripples

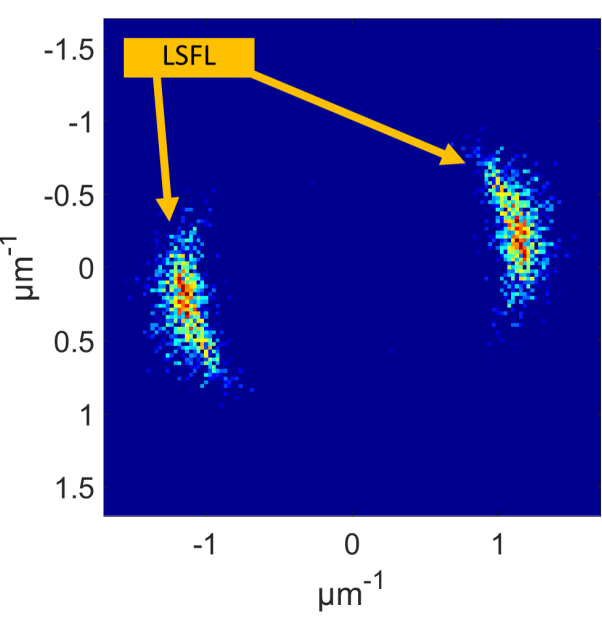

(b) 2D-FFT of LSFL

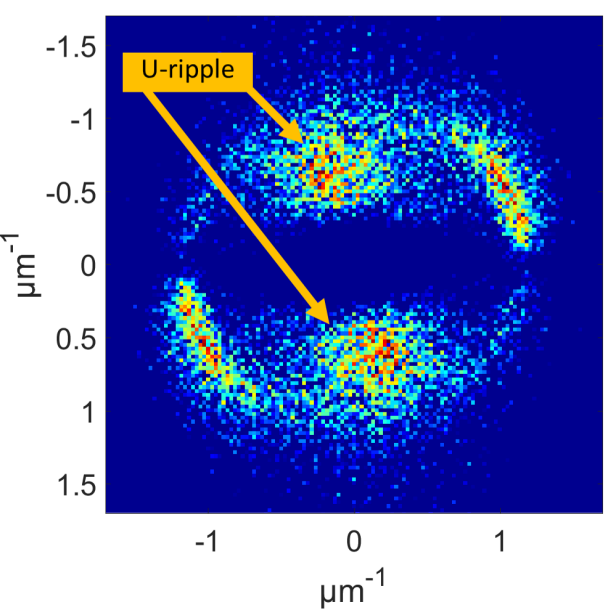

(d) 2D FFT of u-ripples

Figure 3. SEM images of a laser-structured brass surface $(\mathbf{a}, \mathbf{c})$ and corresponding transformed 2D Fourier spectra for the analysis of LSFL and u-ripples $(\mathbf{b}, \mathbf{d})$.

\subsection{Wetting Behavior}

As has been reported, laser-structured surfaces alter the wetting behavior with a temporal evolution of the apparent contact angle over days. Here, we focus on a detailed analysis of the apparent contact angle $\Theta$ as a function of the applied laser fluence and pulse overlap with specific attention on the temporal change in $\Theta$. Figure 4 depicts the apparent contact angle versus time for different fluences between 0.87 and $1.96 \mathrm{~J} / \mathrm{cm}^{2}$. The individual error bars show the influence of the adapted pulse overlap and takes the standard deviation of a threefold measurement into account. The apparent contact angle $\Theta$ was measured directly after the laser irradiation (referred to as Day 1) and on Days 3, 5, 9, 10, and 12. Starting from the initial contact angle of an unstructured surface of $64^{\circ}$, i.e., the hydrophilic range (this contact angle is calculated by Young's equation for a flat surface), for all fluences, the apparent contact angle after laser irradiation and surface structuring initially drops further into the hydrophilic range, a behavior that has been reported by several other groups $[29,34,35]$. During the following days, $\Theta$ continuously increases, indicating a saturation-type behavior reaching values between $100^{\circ}$ and $140^{\circ}$, in accordance with previous observations on several metals [29,34,35]. An explanation for this is given in [29] and [21]. $\mathrm{A} \mathrm{CO}_{2}$ decomposition reaction is triggered by the laser process and generates new non-polar carbon groups on the surface which have a hydrophobic character. It is worth mentioning that, depending on the specific laser structuring parameters, such as 
fluence, pulse overlap, and wavelength, we find that brass saturation is achieved after a period of up to 12 days. With increasing fluence, the apparent contact angle, after the initial drop, increases and leads to a higher apparent contact angle at the end of the observation period of 12 days, shown in Figure 4. Showing the influence of both laser fluence and pulse overlap on the apparent contact angle, Figure 5 details the dependence of these laser structuring parameters on the first and 12th days after irradiation, exemplary for an intermediate fluence of $1.96 \mathrm{~J} / \mathrm{cm}^{2}$. The apparent contact angle on Day 1 significantly decreases with increasing pulse overlap, i.e., increasing track energy, whereas, on Day $12, \Theta$ increases only slightly with pulse overlap. This behavior can, by trend, also be found for the investigated fluences of 0.87 and $1.63 \mathrm{~J} / \mathrm{cm}^{2}$, respectively. In particular, we find that the combination of the highest fluence $\left(1.96 \mathrm{~J} / \mathrm{cm}^{2}\right)$ and the highest specific pulse overlap $(98 \%)$ leads to the smallest contact angle of $8.7^{\circ}$ (Figure 5). In contrast, the combination of the smallest fluence $\left(0.87 \mathrm{~J} / \mathrm{cm}^{2}\right)$ and the smallest specific pulse overlap (92\%) leads to the highest apparent contact angle of $45.3^{\circ}$ on the first day. A similar effect was also reported from Bizi-bandoki et al. [26] for stainless steel and aluminium. At the end of the observation period, i.e., Day 12, all surfaces reached a hydrophobic behavior (Figure 4), and those surfaces with the highest drop of the apparent contact angle after irradiation (Day 1) reached the highest apparent contact angle on the 12th day. In the following section, we will show that this behavior can be attributed to the laser-induced topography, its associated increase in the surface, and changes in the surface chemical composition.
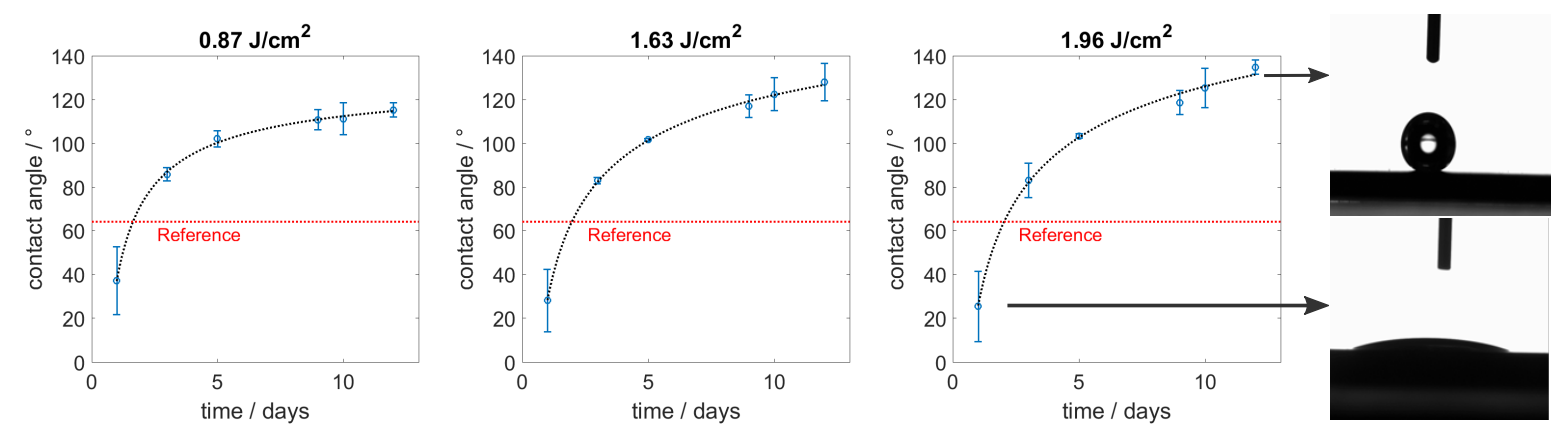

Figure 4. Apparent contact angle in the dependence of time and the applied laser fluence (error bars indicate the standard deviation of 12 daily measured samples). The reference contact angle on flat brass is $64^{\circ}$.

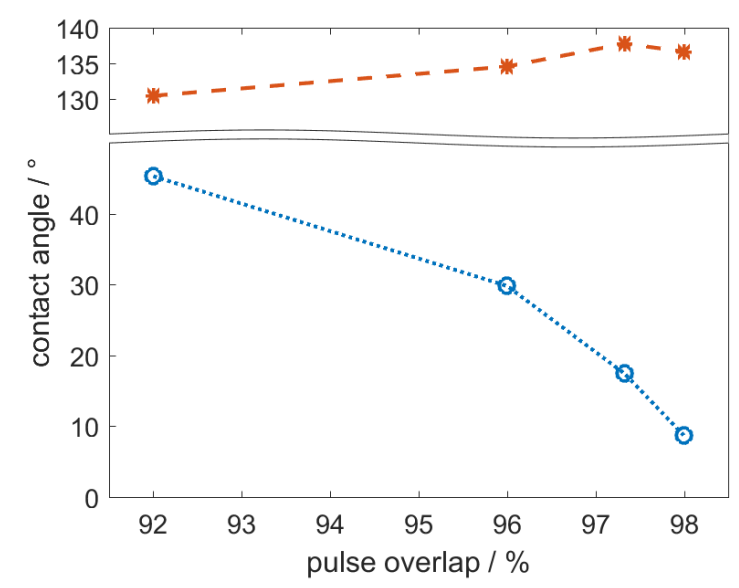

Figure 5. Influence of adapted pulse overlap on the apparent contact angle direct after irradiation (blue) and 12 days later (orange) for a laser fluence of $1.96 \mathrm{~J} / \mathrm{cm}^{2}$. 


\subsection{Structural Characterization}

The influence of the surface roughness upon the apparent contact angle can be explained by the Wenzel state and the Cassie-Baxter state. Regarding Wenzel [30], the inherent wettability of a surface will be enhanced by the surface roughness (see also Equation (1)). Increased roughness will decrease the apparent contact angle on an inherently hydrophilic surface and will increase the apparent contact angle on an inherently hydrophobic surface. This is based on the assumption that the entire surface is wetted, i.e., there is no air gap between the surface and the liquid. The increased roughness, which is induced by laser treatment makes a Wenzel-like wetting more likely. This assumption is in accordance with other research groups [21,26,29]. In terms of the hydrophobic steady state after 12 days, which wetting state is present-the Wenzel, Cassie-Baxter, or Hybrid Cassie-Wenzel model—is still being debated [34].

$$
\cos \left(\Theta_{W}\right)=r \cos \left(\Theta_{Y}\right)
$$

In the following discussion, we focus on the wetting behavior directly after laser treatment. In the Wenzel equation, $r$, generally referred to as the roughness ratio, describes the surface roughness defined by the ratio of the true area of the solid surface to its projected area, i.e., $r$ equals 1 for a perfectly flat surface. However, real surfaces exhibit a certain roughness where $r>1$. According to Equation (1), wetting described by the Wenzel model will always lead to lower apparent contact angles $\Theta_{W}$, as described by Young's equation $\left(\Theta_{Y}\right)$ for perfectly flat surfaces.

Based on this background, our experimental results of a decreased apparent contact angle upon laser irradiation can be assigned to an enlarged surface generated by laser structuring, i.e., the LSFL, u-ripples, and ablation trenches. To estimate $r$, we measured the topography using AFM and 3D optical microscopy and determined the modulation length and depth of LSFL, u-ripples, and ablation trenches. Based on the measured topography (c.f. Figure 6), we derive that the surface can be described by either $|\sin (x)|$ (in case of LSFL) or $\sin (y)$ (in case of u-ripples and ablation trenches) type functions. We, therefore, define the following functions to calculate the real surface area using a numerical approach. Please note that the LSFL are oriented perpendicularly to the u-ripples and ablation trenches (see Figure 2), so the real surface L for LSFL is calculated along the $x$-axis, whereas for u-ripples and ablation tranches $\mathrm{L}$ is calculated along the $y$-axis.

$$
\begin{gathered}
L_{L S F L}=\int_{0}^{\Lambda_{L S F L}} \sqrt{1+\left(\frac{d_{L S F L} \pi \cos \left(\frac{\pi x}{\Lambda_{L S F L}}\right)}{\Lambda_{L S F L}}\right)^{2}} d x \\
L_{\text {u-ripple }}=2 \int_{0}^{\frac{\Lambda_{u-\text {-ripple }}}{2}} \sqrt{1+\left(\frac{d_{u-\text { ripple }} \pi \cos \left(\frac{2 \pi x}{\Lambda_{\text {uripple }}}\right)}{\Lambda_{u-\text {-ripple }}}\right)^{2}} d y \\
L_{\text {ablation }}=2 \int_{0}^{\frac{\Lambda_{\text {ablation }}}{2}} \sqrt{1+\left(\frac{d_{\text {ablation }} \pi \cos \left(\frac{2 \pi x}{\Lambda_{\text {ablation }}}\right)}{\Lambda_{\text {ablation }}}\right)^{2}} d y
\end{gathered}
$$

Here, $L_{L S F L}, L_{u-\text { ripple }}$, and $L_{\text {ablation }}$ denote the real length of the surface in the direction orthogonal to the structures. Dividing them by $\Lambda_{L S F L}, \Lambda_{u \text {-ripple }}$ and $\Lambda_{\text {ablation }}$, respectively, gives the relative extension of the surface by the individual structures. In general, we find that these ratios relate to each other according to

$$
\frac{L_{L S F L}}{\Lambda_{L S F L}}>\frac{L_{u-\text { ripple }}}{\Lambda_{u \text {-ripple }}}>\frac{L_{\text {ablation }}}{\Lambda_{\text {ablation }}} .
$$

For example, for a fluence of $1.96 \mathrm{~J} / \mathrm{cm}^{2}$ and a pulse overlap of $98 \%$, the LSFL leads to an extension of $20.6 \%$, the u-ripple to $9.5 \%$, and the ablation to $1.3 \%$, respectively. The product of these three ratios 
gives the ratio of the true area of the solid surface to its projected area, i.e., $r$ as described in the Wenzel equation. For the above-mentioned example, this results in a roughness ratio $\mathrm{r}$ of 1.34.

As mentioned above, the decrease in the apparent contact angle with laser fluence and pulse overlap can be assigned to the increasing extension of the surface, as generated by the laser structuring. This is qualitatively confirmed and highlighted in Figure 7, showing the correlation of the apparent contact angle and the roughness ratio $r$ versus the pulse overlap. With increasing pulse overlap, i.e., increasing track energy, the roughness ratio increases, while the apparent contact angle decreases accordingly. However, comparing the roughness ratios as determined by the surface topography to the roughness ratios calculated by the Wenzel equation reveals a discrepancy. For example, for the above given example (a fluence of $1.96 \mathrm{~J} / \mathrm{cm}^{2}$ and a pulse overlap of $98 \%$ ) that yields a roughness ratio of $r=1.34$, the apparent contact angle is measured as $8.7^{\circ}$. Using Young's contact angle of $64^{\circ}$ (see above) and the Wenzel equation, the roughness factor is determined to be 2.15. Figure 8 shows the roughness ratios as determined by the surface topography and the roughness ratios calculated by the Wenzel equation using the measured apparent contact angles and Young's contact angle. Apparently, both methods result qualitatively in similar behavior, namely, an increase in $r$ with increasing laser fluence and pulse overlap. However, the roughness ratio as calculated by the Wenzel equation is consistently higher than the roughness ratio determined by the surface topography.

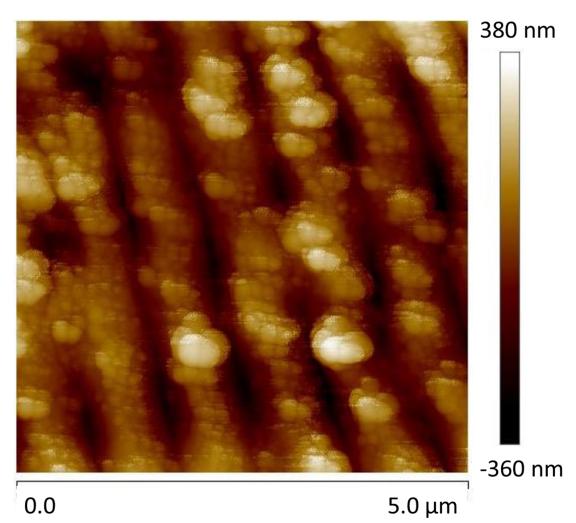

(a) AFM measurement

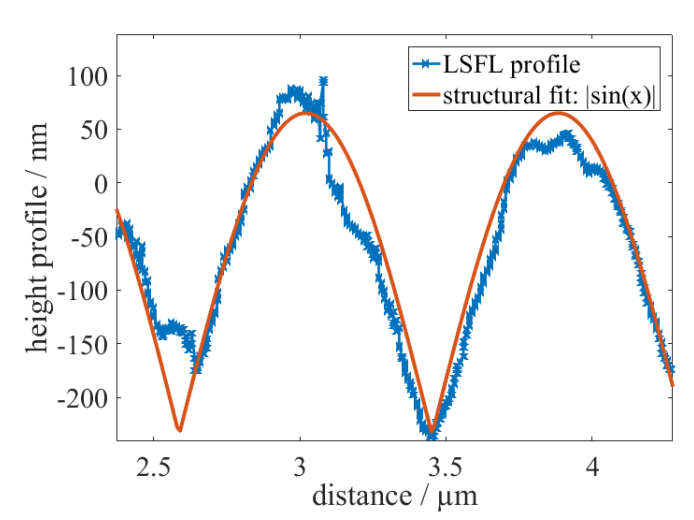

(b) Height profile with structural fit $|\sin (\mathrm{x})|$

Figure 6. AFM measurement of the LSFL structure (a) for a laser fluence of $0.87 \mathrm{~J} / \mathrm{cm}^{2}$ and $99 \%$ overlap. (b) The height profile of the LSFL can be described using a $|\sin (x)|$ function.

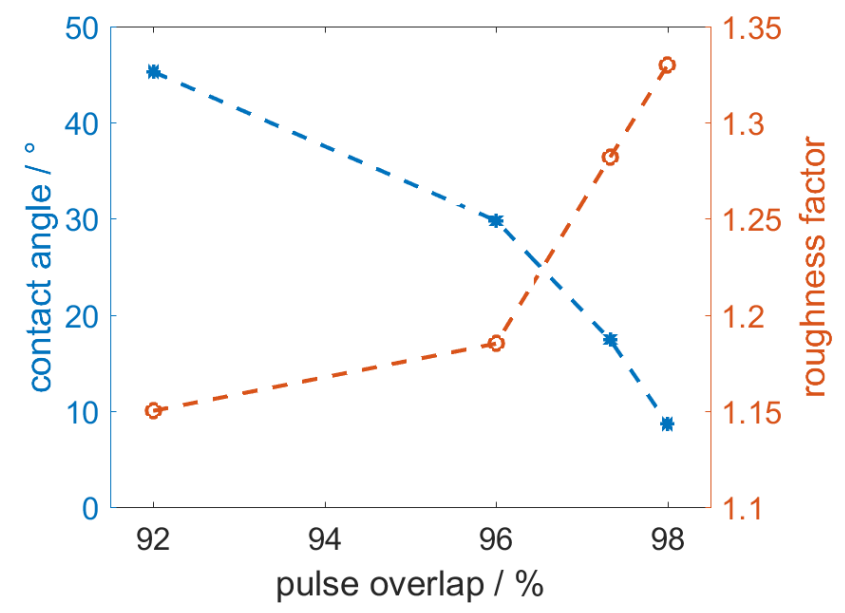

Figure 7. First day apparent contact angle and calculated roughness factor $\mathrm{r}$ for a fluence of $1.96 \mathrm{~J} / \mathrm{cm}^{2}$. 


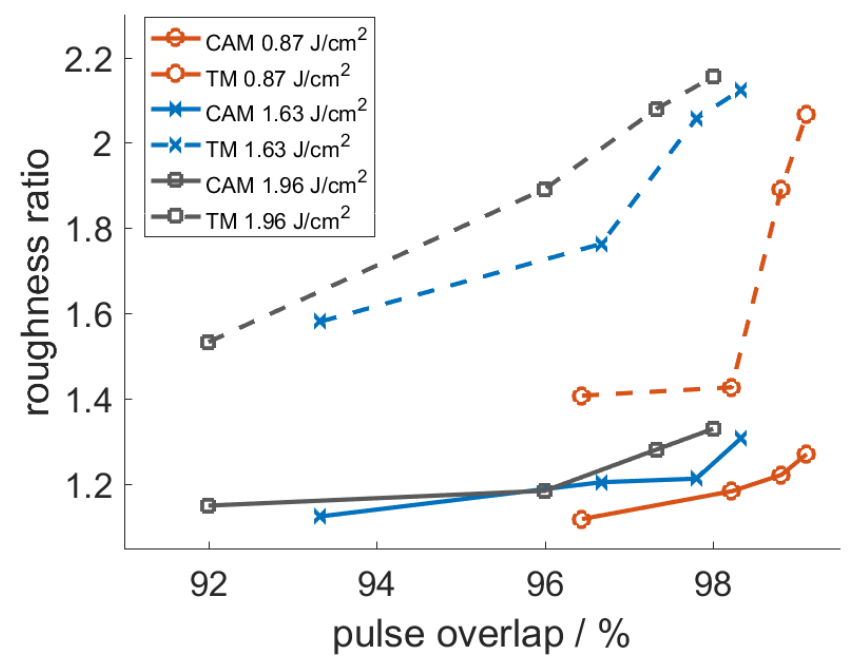

Figure 8. Calculated surface advance based on the topographical measurement (TM, solid lines) and the estimated surface advance based on the apparent contact angle measurement (CAM, dashed lines).

As a consequence, we conclude that the changes in the surface topography are not exclusively responsible for the altered wetting behavior, as it has been assumed by other studies on copper, titanium, stainless steel, and brass [29,34,35]. Thus, a yet unknown surface chemistry effect must be taken into account to further understand the wetting behavior of laser-structured areas. We therefore performed XPS measurements of unstructured reference samples and structured samples on different days after laser treatment. All XPS measurements have been done on previously unmeasured specimens in order to exclude any modifications of the surface chemistry during XPS measurements itself or temporarily storage under high vacuum in the XPS spectrometer.

For the element surface concentration, we find immediately after laser surface structuring that the present carbon concentration strongly decreases from 46.9 to about $22 \%$, while the oxygen concentration increases from about 30 to $52 \%$, respectively, with oxide layers typically being associated with a hydrophilic behavior [29]. These concentrations remain almost unchanged over all days. High resolution detailed XPS carbon spectra (see Figure 9a) additionally reveal a significant increase in oxidic bonds particularly in the form of $\mathrm{O}-\mathrm{C}=\mathrm{O}$ as compared to the reference. In the oxygen spectra (Figure $9 \mathrm{~b}$ ), $\mathrm{C}-\mathrm{O}, \mathrm{Si}-\mathrm{O}, \mathrm{C}=\mathrm{O}$, and metallic oxides can be found for both the reference and laser-treated specimen, but the content of metallic oxides are increased after laser treatment. The small amount of $\mathrm{Si}$ occurring in the measurements is a result of locally introduced $\mathrm{SiC}$ particles by grinding and polishing of the reference surface. Again, these findings remain almost unchanged over days elapsing from the day of laser treatment. We therefore conclude that the changes in element concentration and the binding conditions of carbon and oxygen account for the strong decrease in the apparent contact angle $\Theta$ upon laser structuring. However, as the XPS spectra do not reveal pronounced changes over the following days, the corresponding change in $\Theta$ during the following days remains unclear. 


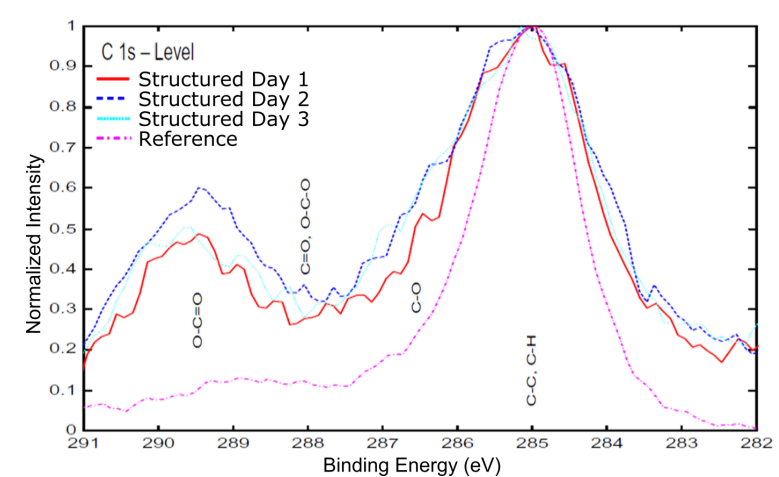

(a)

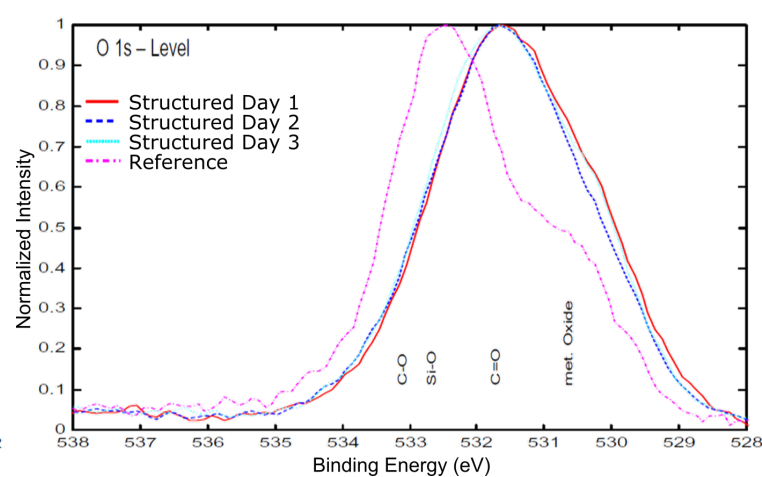

(b)

Figure 9. Normalized intensity resulting from XPS measurement: detailed C-Spectra (a) and O-Spectra (b).

\section{Conclusions}

Using infrared femtosecond laser pulses, large scaled areas completely covered by highly uniformed LIPSS were generated on brass, with these nanostructures being superimposed by microscopic laser ablation trenches and u-ripples. Within a comprehensive experimental study, the wetting behavior of the structured surfaces was investigated as a function of applied laser fluence and pulse overlap, with the temporal evolution of the apparent contact angle measured over several days. The results reveal an initial drop in the apparent contact angle upon laser structuring and a subsequent pronounced increase over the following days. By employing atomic force microscopy and a geometrical model for the laser generated surface topography, the roughness factor given by the Wenzel model was determined and compared with the experimentally determined apparent contact angle, revealing differences that can partly be attributed to changes in the chemical composition on the surface. The results shed light on the wetting behavior of fs-laser-structured surfaces and reveal potential applications for hierarchic nano- and microstructures in surface engineering.

Author Contributions: S.R., S.S. and B.G. conceived and designed the experiments; S.R. and B.G. performed the experiments and analyzed the data; S.R., C.E., and R.H. wrote the paper.

Conflicts of Interest: The authors declare no conflict of interest.

\section{References}

1. Birnbaum, M. Semiconductor Surface Damage Produced by Ruby Lasers. J. Appl. Phys. 1965, 36, 3688-3689. [CrossRef]

2. Bonse, J.; Krüger, J.; Höhm, S.; Rosenfeld, A. Femtosecond laser-induced periodic surface structures. J. Laser Appl. 2012, 24, 042006. [CrossRef]

3. Bonse, J.; Munz, M.; Sturm, H. Structure formation on the surface of indium phosphide irradiated by femtosecond laser pulses. J. Appl. Phys. 2005, 97, 013538. [CrossRef]

4. Bonse, J.; Krüger, J. Pulse number dependence of laser-induced periodic surface structures for femtosecond laser irradiation of silicon. J. Appl. Phys. 2010, 108, 034903. [CrossRef]

5. Zhang, W.; Cheng, G.; Feng, Q. Unclassical ripple patterns in single-crystal silicon produced by femtosecond laser irradiation. Appl. Surf. Sci. 2012, 263, 436-439. [CrossRef]

6. Skolski, J.Z.P. Inhomogeneous Absorption of Laser Radiation: Trigger of LIPSS Formation. J. Laser Micro/Nanoeng. 2013, 8, 1-5. [CrossRef]

7. Bashir, S.; Shahid Rafique, M.; Husinsky, W. Femtosecond laser-induced subwavelength ripples on $\mathrm{Al}$, Si, CaF2 and CR-39. Nucl. Instrum. Methods Phys. Res. Sect. B Beam Int. Mater. Atoms 2012, 275, 1-6. [CrossRef]

8. Dusser, B.; Sagan, Z.; Soder, H.; Faure, N.; Colombier, J.P.; Jourlin, M.; Audouard, E. Controlled nanostructrures formation by ultra fast laser pulses for color marking. Opt. Exp. 2010, 18, 2913-2924. [CrossRef] [PubMed] 
9. Dufft, D.; Rosenfeld, A.; Das, S.K.; Grunwald, R.; Bonse, J. Femtosecond laser-induced periodic surface structures revisited: A comparative study on ZnO. J. Appl. Phys. 2009, 105, 034908. [CrossRef]

10. Seifert, G.; Kaempfe, M.; Syrowatka, F.; Harnagea, C.; Hesse, D.; Graener, H. Self-organized structure formation on the bottom of femtosecond laser ablation craters in glass. Appl. Phys. A 2005, 81, 799-803. [CrossRef]

11. Schwarz, S.; Rung, S.; Hellmann, R. Generation of laser-induced periodic surface structures on transparent material-fused silica. Appl. Phys. Lett. 2016, 108, 181607. [CrossRef]

12. Wallat, K.; Dörr, D.; Le Harzic, R.; Stracke, F.; Sauer, D.; Neumeier, M.; Kovtun, A.; Zimmermann, H.; Epple, M. Cellular reactions toward nanostructured silicon surfaces created by laser ablation. J. Laser Appl. 2012, 24, 042016. [CrossRef]

13. Wang, Z.; Zhao, Q.; Wang, C. Reduction of Friction of Metals Using Laser-Induced Periodic Surface Nanostructures. Micromachines 2015, 6, 1606-1616. [CrossRef]

14. Athanasiou, C.E.; Hongler, M.O.; Bellouard, Y. Unraveling Brittle-Fracture Statistics from Intermittent Patterns Formed During Femtosecond Laser Exposure. Phys. Rev. Appl. 2017, 8, 293. [CrossRef]

15. Madeo, J.; Margiolakis, A.; Zhao, Z.Y.; Hale, P.J.; Man, M.K.L.; Zhao, Q.Z.; Peng, W.; Shi, W.Z.; Dani, K.M. Ultrafast properties of femtosecond-laser-ablated GaAs and its application to terahertz optoelectronics. Opt. Lett. 2015, 40, 3388-3391. [CrossRef] [PubMed]

16. Pazokian, H.; Selimis, A.; Barzin, J.; Jelvani, S.; Mollabashi, M.; Fotakis, C.; Stratakis, E. Tailoring the wetting properties of polymers from highly hydrophilic to superhydrophobic using UV laser pulses. J. Micromechan. Microeng. 2012, 22, 035001. [CrossRef]

17. Ocaña, J.L.; Jagdheesh, R.; García-Ballesteros, J.J. Direct generation of superhydrophobic microstructures in metals by UV laser sources in the nanosecond regime. Adv. Opt. Technol. 2016, 5, 87-93. [CrossRef]

18. Li, B.j.; Li, H.; Huang, L.J.; Ren, N.F.; Kong, X. Femtosecond pulsed laser textured titanium surfaces with stable superhydrophilicity and superhydrophobicity. Appl. Surf. Sci. 2016, 389, 585-593. [CrossRef]

19. Martínez-Calderon, M.; Rodríguez, A.; Dias-Ponte, A.; Morant-Miñana, M.C.; Gómez-Aranzadi, M.; Olaizola, S.M. Femtosecond laser fabrication of highly hydrophobic stainless steel surface with hierarchical structures fabricated by combining ordered microstructures and LIPSS. Appl. Surf. Sci. 2016, 374, 81-89. [CrossRef]

20. Zorba, V.; Persano, L.; Pisignano, D.; Athanassiou, A.; Stratakis, E.; Cingolani, R.; Tzanetakis, P.; Fotakis, C. Making silicon hydrophobic: Wettability control by two-lengthscale simultaneous patterning with femtosecond laser irradiation. Nanotechnology 2006, 17, 3234-3238. [CrossRef]

21. Ngo, C.V.; Chun, D.M. Fast wettability transition from hydrophilic to superhydrophobic laser-textured stainless steel surfaces under low-temperature annealing. Appl. Surf. Sci. 2017, 409, 232-240. [CrossRef]

22. Yang, Z.; Tian, Y.L.; Yang, C.J.; Wang, F.J.; Liu, X.P. Modification of wetting property of Inconel 718 surface by nanosecond laser texturing. Appl. Surf. Sci. 2017, 414, 313-324. [CrossRef]

23. Rajab, F.H.; Liu, Z.; Li, L. Production of stable superhydrophilic surfaces on 316L steel by simultaneous laser texturing and $\mathrm{SiO} 2$ deposition. Appl. Surf. Sci. 2018, 427, 1135-1145. [CrossRef]

24. Luo, B.H.; Shum, P.W.; Zhou, Z.F.; Li, K.Y. Preparation of hydrophobic surface on steel by patterning using laser ablation process. Surf. Coat. Technol. 2010, 204, 1180-1185. [CrossRef]

25. Martínez-Calderon, M.; Rodrígueza, A.; Diasa, A.; Gómez-Aranzadia, M.; Olaizolaa, S.M. Femtosecond laser manufacturing of highly hydrophobic hierarchical structures fabricated by combining surface microstructures and LIPSS. In Proceedings of the Lasers in Manufacturing Conference; Wissenschaftliche Gesellschaft Lasertechnik e.V.: Erlangen, Germany, 2015.

26. Bizi-bandoki, P.; Valette, S.; Audouard, E.; Benayoun, S. Time dependency of the hydrophilicity and hydrophobicity of metallic alloys subjected to femtosecond laser irradiations. Appl. Surf. Sci. 2013, 273, 399-407. [CrossRef]

27. Raimbault, O.; Benayoun, S.; Anselme, K.; Mauclair, C.; Bourgade, T.; Kietzig, A.M.; Girard-Lauriault, P.L.; Valette, S.; Donnet, C. The effects of femtosecond laser-textured Ti-6Al-4V on wettability and cell response. Mater. Sci. Eng C Mater. Biol. Appl. 2016, 69, 311-320. [CrossRef] [PubMed]

28. Ta, D.V.; Dunn, A.; Wasley, T.J.; Kay, R.W.; Stringer, J.; Smith, P.J.; Connaughton, C.; Shephard, J.D. Nanosecond laser textured superhydrophobic metallic surfaces and their chemical sensing applications. Appl. Surf. Sci. 2015, 357, 248-254. [CrossRef] 
29. Kietzig, A.M.; Negar Mirvakili, M.; Kamal, S.; Englezos, P.; Hatzikiriakos, S.G. Laser-patterned super-hydrophobic pure metallic substrates: Cassie to wenzel wetting transitions. J. Adhes. Sci. Technol. 2011, $25,2789-2809$.

30. Wenzel, R.N. Surface Roughness and Contact Angle. J. Phys. Colloid Chem. 1949, 53, 1466-1467. [CrossRef]

31. Okamuro, K.; Hashida, M.; Miyasaka, Y.; Ikuta, Y.; Tokita, S.; Sakabe, S. Laser fluence dependence of periodic grating structures formed on metal surfaces under femtosecond laser pulse irradiation. Phys. Rev. B 2010, 82. [CrossRef]

32. Golosov, E.V.; Emel'yanov, V.I.; Ionin, A.A.; Kolobov, Y.R.; Kudryashov, S.I.; Ligachev, A.E.; Novoselov, Y.N.; Seleznev, L.V.; Sinitsyn, D.V. Femtosecond laser writing of subwave one-dimensional quasiperiodic nanostructures on a titanium surface. JETP Lett. 2009, 90, 107-110. [CrossRef]

33. Yu, J.; Lu, Y. Laser-induced ripple structures on Ni-P substrates. Appl. Surf. Sci. 1999, 148, $248-252$. [CrossRef]

34. Van Ta, D.; Dunn, A.; Wasley, T.J.; Li, J.; Kay, R.W.; Stringer, J.; Smith, P.J.; Esenturk, E.; Connaughton, C.; Shephard, J.D. Laser textured surface gradients. Appl. Surf. Sci. 2016, 371, 583-589.

35. Van Ta, D.; Dunn, A.; Wasley, T.J.; Li, J.; Kay, R.W.; Stringer, J.; Smith, P.J.; Esenturk, E.; Connaughton, C.; Shephard, J.D. Laser textured superhydrophobic surfaces and their applications for homogeneous spot deposition. Appl. Surf. Sci. 2016, 365, 153-159.

(C) 2018 by the authors. Licensee MDPI, Basel, Switzerland. This article is an open access article distributed under the terms and conditions of the Creative Commons Attribution (CC BY) license (http://creativecommons.org/licenses/by/4.0/). 